\title{
A simple direction-sensitive circuit
}

\author{
ALLISTON K. REID \\ Duke University, Durham, North Carolina 27706
}

A circuit is described that determines the direction of travel by the order in which a subject interrupts two light beams. Its operation in a two-chambered apparatus and in a multichambered apparatus is demonstrated to allow mutually exclusive and exhaustive location description.

Many questions regarding animal behavior require information about the direction of movement or the location of the animal in a multichamber apparatus. The circuit depicted in Figure 1 a determines the direction of travel by the order in which the subject interrupts two light beams. The simplest application of the direction-sensitive circuit is in a two-chamber apparatus, depicted in Figure $1 \mathrm{~b}$. The two outputs in Figure 1a (Relays A and B) represent the two possible locations of the subject, only one of which can operate at any given time. The doorway between the two

This research was supported by Grant BNS 76-23182 from the National Science Foundation to Duke University, J. E. R. Staddon, Principal Investigator. chambers contains two photocells separated by about $2 \mathrm{~cm}$. As the subject travels from Chamber A to Chamber $B$, the following changes occur: (1) The light beam illuminating Photocell $\mathrm{P}_{\mathrm{A}}$ (the photocell closer to the subject) is interrupted, with no resulting change in output (the circuit should already signal that the subject is in Chamber A). (2) The light beam illuminating Photocell $\mathrm{P}_{\mathrm{B}}$ (farther from the subject) is interrupted, while the light beam normally illuminating Photocell $\mathbf{P}_{\mathbf{A}}$ remains interrupted. Still, no change in output conditions results. (3) The light beam focused at Photocell $\mathrm{P}_{\mathrm{A}}$ resumes illumination of the photocell, resulting in an output change: Relay $B$ is now latched, and Relay A released. (4) The light beam resumes illumination of Photocell $\mathrm{P}_{\mathrm{B}}$ once the subject (a) 7413
7480
7474

$2 \mathrm{~N} 3994$

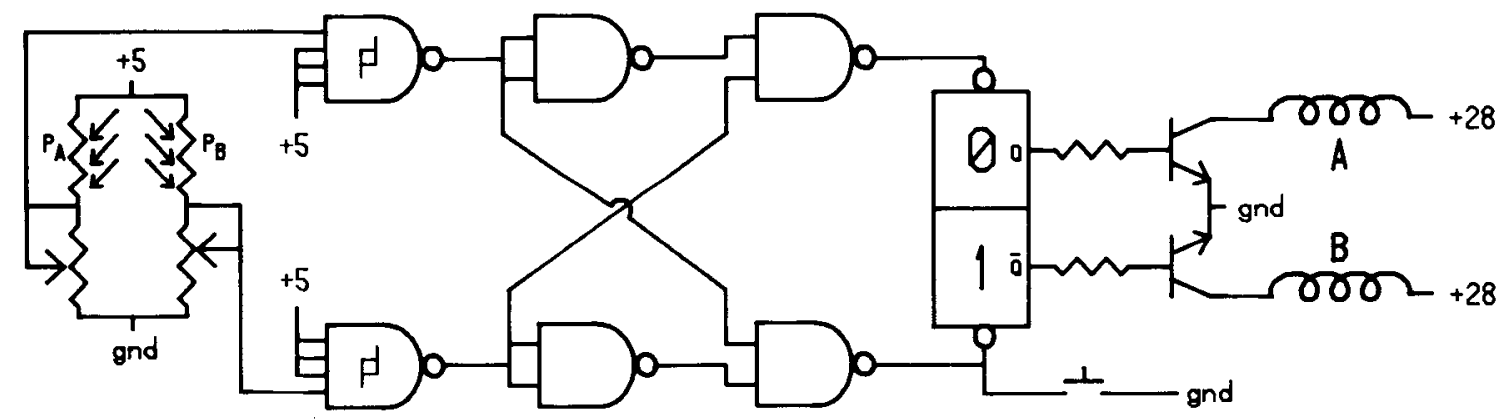

(b)

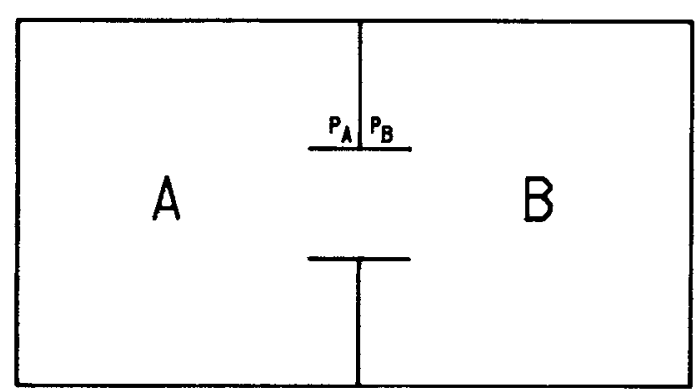

Figure 1. (a) A simple direction-sensitive circuit. The determination of direction is dependent upon the order of interruption of illumination on two photocells, $P_{A}$ and $P_{B^{-}}$(b) $A$ two-chamber apparatus with the two photocells in the doorway (top view). 
completely passes the doorway, with no resulting change in output.

Note that "nosing" (breaking only one light beam by partially entering the doorway) has no effect on the output. The circuit signals a change in location only when the subject reestablishes the first light beam it interrupts by passing completely through the doorway. However, since the output does depend on the order of input, the doorway must be constructed so that the proper order always occurs. It is usually sufficient to restrict the size of the doorway.

The circuit operates on the following principle: Interruption of illumination on Photocell $\mathrm{P}_{\mathrm{A}}$ decreases the input voltage into the NAND Schmitt trigger, resulting in a high-voltage output. The first NAND gate inverts the digital signal, producing a high output from the second NAND gate in the series. Since the flip-flop has inverting inputs (the "clear" input in this case), its output is not altered by the high input. This situation occurs independently of the state of Photocell $P_{B}$. No change in output occurs with either light beam interruption, since both result in a high input to the flip-flop. When illumination of a photocell is reinstated, the high-voltage input to a Schmitt trigger results in a low output to an inverting flip-flop input. If the low input to the flip-flop activates a different input than that last activated, a change in output occurs. If the input activates the input last activated, no change occurs. Thus, the reinstatement of illumination on either photocell results in an output change only if that photocell was not the last one reinstated the last time either light beam was interrupted.

The location of the subject in an apparatus with more than two chambers may be determined by building a circuit for each doorway and connecting the " $Q$ " output of each flip-flop to the preset input of the flip-flops in all the other circuits. In this configuration, each circuit acts as a check on the other circuits, so that only one location may be signaled at any time. The " $\bar{Q}$ " output is not used in this situation.

To determine the amount of time taken for the subject to pass through the doorway, the user may simply insert an OR gate after the two Schmitt triggers and use it as a third output. This will indicate the amount of time either (and both) of the light beams is interrupted. An AND gate in that position indicates the time that both light beams are interrupted concurrently.

The circuit must be preset each time a new subject is placed into the apparatus, so that the output will correctly represent the starting location of the subject. A push button (Figure 1a) wired to the preset of the flip-flop allows initialization of the circuit; this signals that the subject is starting the session in Chamber A. Wiring the push button to the "clear" of the flip-flop signals that the subject is starting in Chamber B.

The value of the variable resistors is dependent on the type of photocell used. With most cadmium sulfide cells, a resistance of about $10 \mathrm{kohms}$ will probably cover a broad enough range for operation. The photocells may be replaced by infrared detectors or phototransistors for differential sensitivity to different light conditions.

The outputs of the flip-flops depicted in Figure 1a are used to drive relays; however, elimination of the two relays, transistors, and resistors allows it to drive other TTL circuitry.

In summary, the circuit depicted in Figure la determines the direction of travel by the order in which the subject interrupts two light beams. This circuit is essentially foolproof and overcomes problems inherent in other circuits resulting from "uncooperative movements," such as lying down or turning around in front of the sensors. It also establishes an objective criterion for determining the time of transition between areas in the apparatus. Thus, it allows for mutually exclusive and, frequently, exhaustive location description.

The circuit contains two photocells, two variable resistors, two resistors, three ICs, two transistors, two relays, and one push-button switch, for a total cost of approximately $\$ 10$.

(Received for publication June 28, 1978; revision accepted October 5,1978 .) 